\title{
Biotic and anthropogenic forces rival climatic/abiotic factors in determining global plant population growth and fitness
}

\author{
William F. Morris ${ }^{a, 1}$, Johan Ehrlén ${ }^{b}$, Johan P. Dahlgren ${ }^{c, d}$, Alexander K. Loomis ${ }^{a}$, and Allison M. Louthan ${ }^{a}$ \\ aDepartment of Biology, Duke University, Durham, NC 27708; 'bepartment of Ecology, Environment and Plant Sciences, Stockholm University, SE-106 91 \\ Stockholm, Sweden; 'Department of Biology, University of Southern Denmark, DK-5230 Odense M, Denmark; and Interdisciplinary Centre on Population \\ Dynamics, University of Southern Denmark, DK-5230 Odense M, Denmark
}

Edited by Alan Hastings, University of California, Davis, CA, and approved December 5, 2019 (received for review October 22, 2019)

Multiple, simultaneous environmental changes, in climatic/abiotic factors, interacting species, and direct human influences, are impacting natural populations and thus biodiversity, ecosystem services, and evolutionary trajectories. Determining whether the magnitudes of the population impacts of abiotic, biotic, and anthropogenic drivers differ, accounting for their direct effects and effects mediated through other drivers, would allow us to better predict population fates and design mitigation strategies. We compiled 644 paired values of the population growth rate $(\lambda)$ from high and low levels of an identified driver from demographic studies of terrestrial plants. Among abiotic drivers, natural disturbance (not climate), and among biotic drivers, interactions with neighboring plants had the strongest effects on $\lambda$. However, when drivers were combined into the 3 main types, their average effects on $\lambda$ did not differ. For the subset of studies that measured both the average and variability of the driver, $\lambda$ was marginally more sensitive to 1 SD of change in abiotic drivers relative to biotic drivers, but sensitivity to biotic drivers was still substantial. Similar impact magnitudes for abiotic/biotic/anthropogenic drivers hold for plants of different growth forms, for different latitudinal zones, and for biomes characterized by harsher or milder abiotic conditions, suggesting that all 3 drivers have equivalent impacts across a variety of contexts. Thus, the best available information about the integrated effects of drivers on all demographic rates provides no justification for ignoring drivers of any of these 3 types when projecting ecological and evolutionary responses of populations and of biodiversity to environmental changes.

population growth rate | climate change | environmental driver | species interactions $\mid$ anthropogenic impacts

Derate erhaps the greatest challenge ecologists now face is to accurately predict how multiple, simultaneous, and ongoing environmental changes will impact the abundances and geographical distributions of species, and thus global patterns of biodiversity and the ecosystem services they provide to humans $(1,2)$. Ecologists know that changes in abiotic conditions (e.g., due to climate change), in biotic interactions (e.g., due to species introductions), and in direct human impacts (e.g., due to harvesting) can all impact populations. However, some of these changes may be more impactful than others. Knowing which (if any) of these changes are likely to be more important would allow us to focus on those factors when predicting population responses and when designing mitigation strategies to reduce the most detrimental impacts. Even though one of these factors could impact populations via changes in another factor (e.g., climate change altering the strength of species interactions), knowing which factor has the greatest total impact (including both direct impacts and indirect impacts mediated through other factors) would be valuable.

We currently lack a comprehensive assessment of the relative impacts of different types of drivers (i.e., environmental factors) on a single metric of population performance across multiple species in any major taxon. To redress this lack, we undertook an assessment of the relative impacts of abiotic, biotic, and anthropogenic drivers on the per-capita growth rate of plant populations. Percapita population growth rate $(\lambda)$ is a metric of change in abundance over time, and thus partly determines geographic distributions (3-5). It is also a measure of mean absolute fitness, or of absolute fitness when measured for individual genotypes $(6,7)$. For perennial organisms with overlapping generations, structured population models quantify $\lambda$ by integrating multiple demographic processes and life stages (8). Hundreds of such models have now been constructed that measure the impacts of myriad drivers on plant population growth, but to our knowledge no previous syntheses have exploited this knowledge base to ask which drivers most affect $\lambda$, and thus which are essential and which (if any) can be ignored when predicting the consequences of future environmental changes for ecological and evolutionary change. Here, we focus on terrestrial plants. As they are the primary producers in terrestrial ecosystems, which include some of the most productive ones on Earth, factors that affect fitness and population dynamics in terrestrial plants will likely have knock-on effects on other species in the ecosystem, as well as on feedbacks (e.g., through carbon sequestration) to global climate. We collected data from 207 published studies that yielded 644 comparisons of $\lambda$ values at different levels of multiple drivers for 208 terrestrial plant species from 72 families (Dataset S1) (9).

\section{Significance}

Knowing which of multiple environmental factors (climate, other species, humans, etc.) most strongly affect wild plants and animals could focus our attention on the future environmental changes most likely to influence biodiversity. However, we find that abiotic, biotic, and human influences on plant populations are of similar strengths, for different kinds of plants and in multiple locations and environments. The effects of these factors on plant evolution are also likely to be similar. Thus, there is unlikely to be a shortcut to considering all of these factors when predicting the future ecological and evolutionary responses of species and of biodiversity to environmental changes.

Author contributions: W.F.M., J.E., J.P.D., A.K.L., and A.M.L. designed research; W.F.M., J.E., J.P.D., A.K.L., and A.M.L. performed research; W.F.M. analyzed data; and W.F.M., J.E., J.P.D., A.K.L., and A.M.L. wrote the paper.

The authors declare no competing interest.

This article is a PNAS Direct Submission.

This open access article is distributed under Creative Commons Attribution-NonCommercialNoDerivatives License 4.0 (CC BY-NC-ND).

Data deposition: All of the data extracted from the published studies that were used to perform the analyses in this paper are available in SI Appendix, Tables S1 and S2, and are archived on Dryad (DOI: 10.5061/dryad.95x69p8fm).

${ }^{1}$ To whom correspondence may be addressed. Email: wfmorris@duke.edu.

This article contains supporting information online at https://www.pnas.org/lookup/suppl/ doi:10.1073/pnas.1918363117/-/DCSupplemental.

First published December 30, 2019. 
We classified the drivers identified by the authors of the studies into the 3 main types, each containing 3 subtypes. Abiotic drivers included climate (temperature, precipitation, soil moisture variation due to climate, snow cover or amount, and growing season length), nonclimate abiotic drivers (including soil nutrients and $\mathrm{pH}$, soil texture, ground slope, litter level, site-level moisture not directly related to climate, and $\mathrm{CO}_{2}$ concentration), and disturbance (which included infrequent natural events: fires, hurricanes, flooding, and animal disturbance but not consumption). Biotic drivers included interactions with neighboring plants (which could be competing with or facilitating the focal species), natural enemies (including herbivores and-rarely-pathogens), and mutualists (pollinators, seed dispersers, endophytes, and defensive ants). Anthropogenic drivers included harvesting (of plant parts or entire individuals), grazing by domesticated herbivores and mowing, and land use (including habitat fragmentation and human disturbance).

The relative impacts of different drivers could differ based on plant growth form or local environmental conditions. If, for example, abiotic drivers were found to exert stronger effects on average than do biotic factors on herbaceous but not woody plants, we might be more justified in focusing on abiotic changes when projecting future abundances and distributions of herbaceous (but not woody) plants. Abiotic drivers could also be more impactful at high latitudes or under harsher environmental conditions $(10,11)$, which could give an a priori expectation of which factors deserve more attention depending on species or location. Because our database included plants of different growth forms, from different latitudes, and in different biomes, we were able to evaluate how the relative strengths of different driver types might vary.

One challenge in comparing the impacts of different driver types is that many studies that have quantified $\lambda$ at relatively high vs. relatively low levels of a driver (e.g., in wetter vs. drier locations or years, or at ambient levels of herbivores vs. with herbivores excluded) did not actually measure the levels of the drivers (3). While these studies provide valuable information about impacts of different drivers on $\lambda$, we cannot be certain whether the differences in driver levels are comparable across different types of drivers. Therefore, we performed our analysis at 2 levels. At the broader level, we used all 644 paired values of $\lambda$ in our database to assess whether the difference in $\lambda$ at relatively high vs. relatively low levels of the driver differed between driver types, plant growth forms, geographical regions, and environmental severity levels. At the more focused level, we used the subset of $\lambda$ comparisons for which driver levels had been measured to ask: did the difference in driver levels, relative to the natural range of variation of each driver, differ between different types of drivers, and for a comparable change in driver levels, did the impacts on $\lambda$ differ for different types of drivers?

\section{Results}

We first measured the impact of drivers on population growth rates in the full dataset using the absolute value of the log response ratio:

$$
|L|=\left|\log \left(\lambda_{\text {high }} / \lambda_{\text {low }}\right)\right|,
$$

where $\lambda_{\text {high }}$ and $\lambda_{\text {low }}$ are the population growth rates at relatively high and relatively low levels of the driver, respectively. We use the absolute value as we are interested in comparing the magnitudes of the effects of drivers that have negative (e.g., herbivory or competition) and positive (e.g., pollination or facilitation) effects on $\lambda$ (for more details, see Methods).

Driver subtypes differed significantly in their effects on $\lambda$ (Fig. $1 A$; weighted linear mixed model on log-transformed $|L|$ values; analysis of deviance $\left.\chi^{2}=42.44, \mathrm{df}=8, P=1.1 \times 10^{-6}\right)$. Disturbance and interactions with neighboring plants had the largest effects on $\lambda$. However, if we combine driver subtypes into the 3 main driver
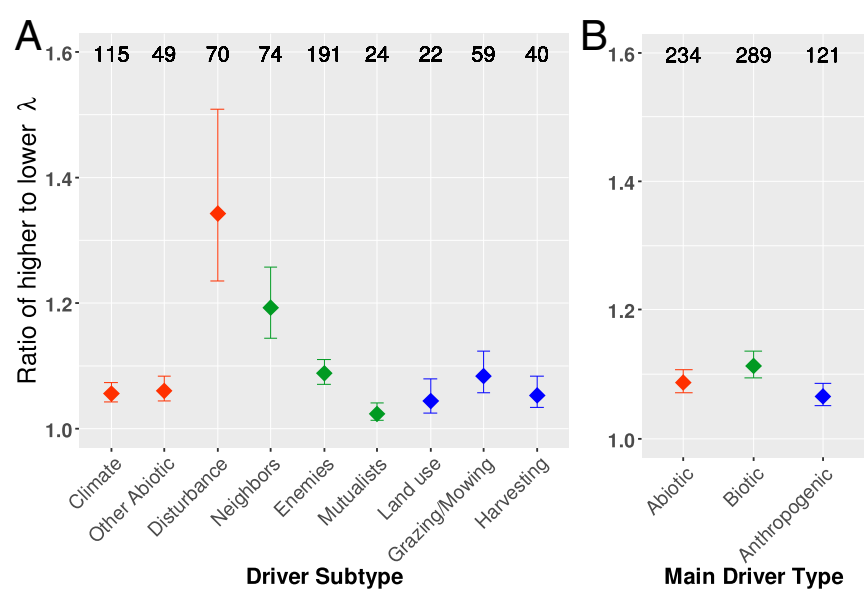

Fig. 1. Effects of different types of drivers on plant population growth rate, $\lambda$, measured as the ratio of the higher to the lower $\lambda$ value at relatively high and relatively low levels of the driver. Points and error bars show the best estimates of the backtransformed coefficients from the linear mixed model $\pm 1 \mathrm{SE}$. $(A)$ Drivers are classified into 9 subtypes (see text for descrip tions of specific drivers). (B) The driver subtypes in $A$ are grouped into 3 main driver types: red, abiotic; green, biotic; and blue, anthropogenic. Numerals at top indicate the number of $\lambda$ pairs for each driver type.

types, there is no significant difference between the effects of abiotic, biotic, and anthropogenic drivers (Fig. $1 B ; \chi^{2}=3.17$, $\mathrm{df}=2, P=0.21$ ).

The lack of a difference between main driver types could have arisen if drivers with weaker effects on $\lambda$ varied over a larger range of values in the studies in our database. In particular, studies of abiotic drivers typically exploited variation in the driver over space or time to identify relatively high and low driver levels, whereas studies of biotic drivers were more likely to manipulate the driver either experimentally (e.g., using cages to entirely exclude herbivores) or "virtually" (e.g., modifying the structured population model to eliminate all measured herbivore effects on plant demographic rates), either of which could in principle have caused a larger difference in driver levels (but see SI Appendix). To assess the range of driver values, we extracted from our database all cases $(n=105)$ in which the original study provided the levels of the driver corresponding to the values of $\lambda_{\text {high }}$ and $\lambda_{\text {low }}$ as well as the mean and SD of the driver across space and/or time. These included 62 cases of abiotic and 43 cases of biotic drivers ( 3 additional cases of anthropogenic drivers provided too little data to be included in the analysis). We first verified that, as in the analysis of the full dataset, $|L|$ did not differ between abiotic and biotic drivers $\left(\chi^{2}=0.085, \mathrm{df}=1, P=0.77\right)$. We next compared the deviation, measured in SDs away from the driver mean, of the higher or lower driver level (whichever deviation was larger) for abiotic vs. biotic drivers, and found that the cases of biotic drivers in our data subset had on average a greater deviation than did the cases of abiotic drivers (Fig. $2 A ; \chi^{2}=9.49, \mathrm{df}=1, P=0.0021$; see SI Appendix for more on the analysis). Thus relative to the natural variation in the drivers, the range of biotic drivers in our data subset was greater.

We then asked: if abiotic and biotic drivers in the data subset do not differ in $|L|$, but biotic drivers have a larger relative range (measured in SD units), is $\lambda$ less sensitive to biotic drivers? For each pair of $\lambda$ values, we computed the following:

$$
|S|=\left|\frac{\left(\lambda_{\text {high }}-\lambda_{\text {low }}\right)}{\left(d_{\text {high }}-d_{\text {low }}\right) / S D_{d}}\right|,
$$

where $d_{\text {high }}, d_{\text {low }}$, and $S D_{d}$ are the driver values that produced the high and low $\lambda$ values and the SD of the driver, respectively. 

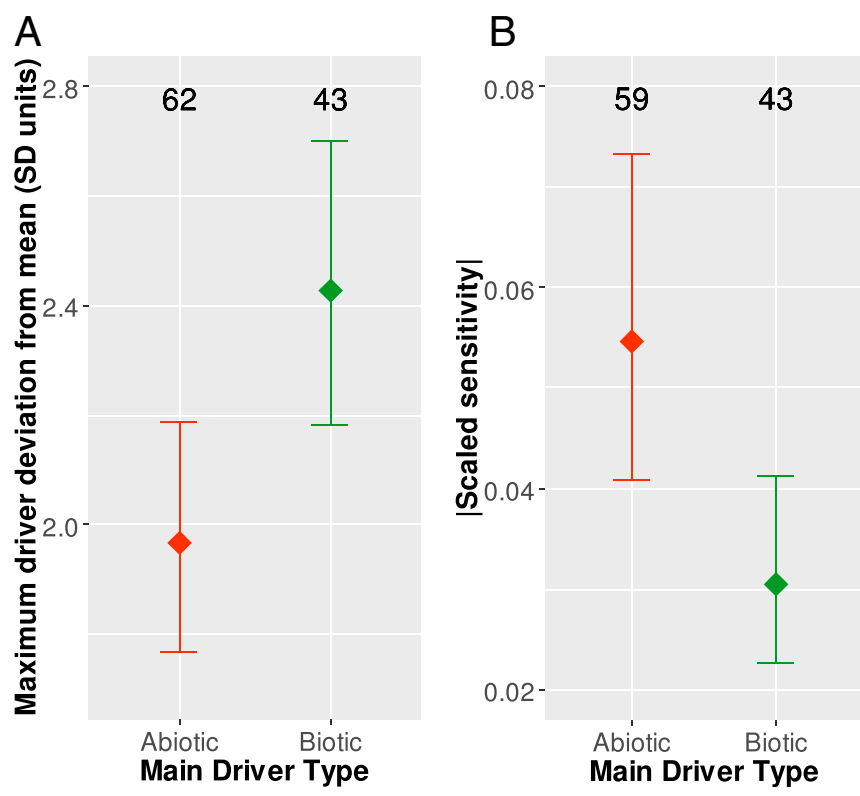

Fig. 2. (A) In the data subset that included measures of driver means and SDs, biotic drivers varied more (in SD units) than abiotic drivers. (B) The scaled sensitivity of the population growth rate (Eq. 2) to abiotic drivers is marginally significantly higher when low outliers for abiotic drivers are omitted. Points and error bars show the best estimates of the backtransformed coefficients from the linear mixed model $\pm 1 \mathrm{SE}$.

Because the denominator of $|S|$ is the difference in driver levels in SD units, $|S|$ serves to compare the sensitivities of the population growth rate to drivers that vary over different scales (hence, we refer to $|S|$ as a "scaled sensitivity"). As for $L$, we use the absolute value as we are interested in the magnitude rather than the direction of the driver's effects on $\lambda$. If we delete $3|S|$ values for abiotic drivers judged to be low outliers, then the sensitivity of $\lambda$ to abiotic drivers is marginally significantly higher than to biotic drivers (Fig. $2 B ; \chi^{2}=3.18, \mathrm{df}=1, P=0.075$ ). The average value of $|S|$ for biotic drivers is $55.9 \%$ of the average value for abiotic drivers; if the low $|S|$ outliers for abiotic drivers are included, this value increases to $67.9 \%$ (and the difference in $|S|$ between abiotic and biotic drivers is no longer even marginally significant). Thus, the best estimate is that $1 \mathrm{SD}$ of change in a biotic driver causes on average one-half to two-thirds as much change in population growth as the same relative change in an abiotic driver, although this difference is not strongly statistically supported.

Given that most studies did not quantify driver levels, we cannot assess whether the greater difference in driver levels and the lower sensitivity of $\lambda$ to biotic drivers holds for the full dataset. However, we can use the full dataset to assess whether $|L|$ differs with plant growth form, latitude, or degree of environmental severity. Plant species differed in the effects of the main driver types based on their growth form (Fig. 3; driver type $\times$ growth form interaction: $\chi^{2}=9.12$, df $=2, P=0.010$ ), with woody plants showing notably less impact of anthropogenic drivers. However, when we exclude anthropogenic drivers, woody and herbaceous plants do not differ significantly in the impacts of abiotic vs. biotic drivers (2-way interaction: $\chi^{2}=2.73, \mathrm{df}=1, P=0.098$; additive model main effect of driver type: $\chi^{2}=0.55$, df $=1, P=0.45$ ), but herbaceous plants were marginally more strongly influenced by all drivers on average than were woody plants (additive model main effect of growth form: $\chi^{2}=3.53$, df $=1, P=0.060$ ).

The effect of main driver type did not differ between latitudinal zones (Fig. 4; driver type $\times$ latitudinal zone interaction: $\left.\chi^{2}=2.85, \mathrm{df}=4, P=0.58\right)$; this remains true $\left(\chi^{2}=0.95, \mathrm{df}=2\right.$, $P=0.62)$ when anthropogenic drivers are excluded. In an additive model, driver types do not differ in effect magnitudes $\left(\chi^{2}=1.26\right.$, $\mathrm{df}=2, P=0.53)$, but there is a significant effect of latitudinal zone $\left(\chi^{2}=7.24, \mathrm{df}=2, P=0.023\right)$; plants in intermediate and higher latitudes were more affected on average by drivers of all types.

We used average net primary production (NPP) as an indicator of the degree of harshness of the abiotic environment in different biomes (Methods). The magnitudes of the effects of the main driver types also did not differ between high and low NPP biomes (Fig. 5); the driver type $\times$ biome type interaction was not significant $\left(\chi^{2}=1.06, \mathrm{df}=2, P=0.59\right)$, and in an additive model, driver type was not significant $\left(\chi^{2}=3.62\right.$, $\left.\mathrm{df}=2, P=0.16\right)$. However, drivers of all types had a higher average effect in low NPP biomes than in high NPP biomes $\left(\chi^{2}=16.58, \mathrm{df}=1, P=4.6 \mathrm{e}-5\right)$.

\section{Discussion}

Our analysis of the best available data shows that there is no one main type of driver (abiotic, biotic, or anthropogenic) that has overwhelmingly stronger effects on plant population growth and fitness relative to the others. Using a portion of the full dataset, we did find that $\lambda$ was marginally significantly less sensitive on average to changes in biotic relative to abiotic drivers (when changes are measured in units of SDs; Fig. $2 B$ ), but this difference is weakly supported and deserves to be examined further when more data become available. The best estimates of the average scaled sensitivities suggest that biotic changes will be more than one-half as influential as abiotic changes of comparable magnitude, and thus worthy of consideration. Our conclusion based on analysis of the full dataset that changes in abiotic, biotic, and anthropogenic drivers all need to be considered largely holds for plants with different growth forms, for different latitudinal zones, and for both harsher and more benign abiotic environments (as indicated by biome-level NPP). The generality of this result has 4 important ecological and evolutionary implications.

First, when predicting how the distributions and abundances of plant species will respond to climate change, considering only the effects of changes in climatic drivers is not likely to yield accurate predictions. Despite widespread recognition that interspecific

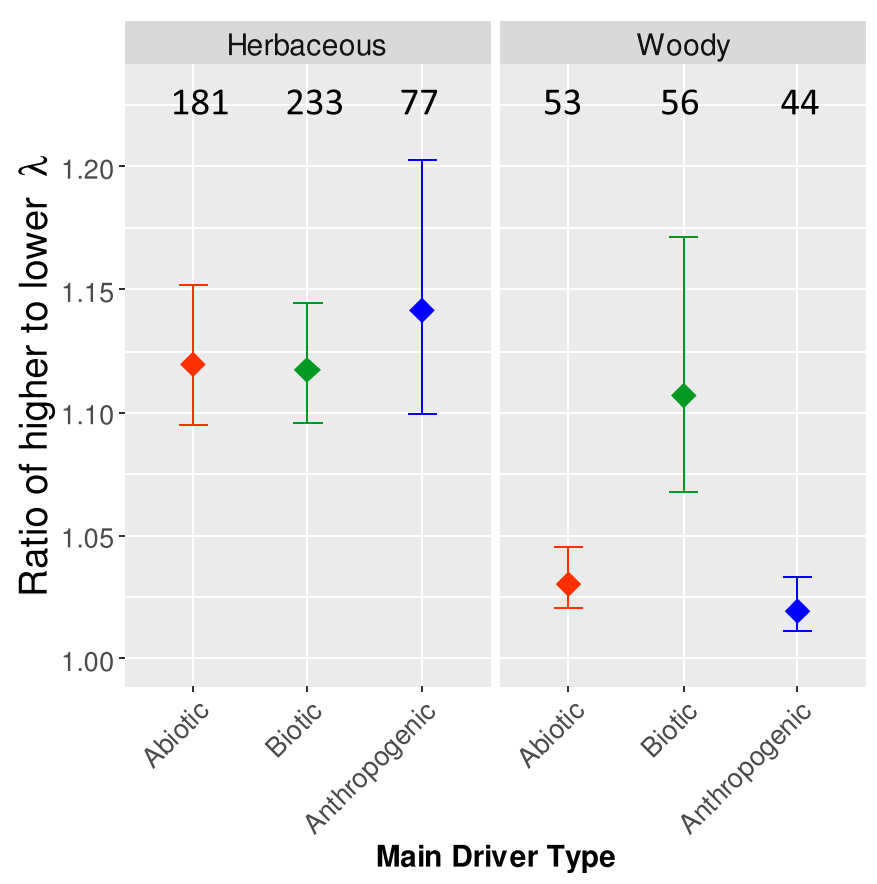

Fig. 3. Effects of main driver types on population growth depended on plant growth form (herbaceous vs. woody); points, error bars, and numerals at Top as in Fig. 1. 


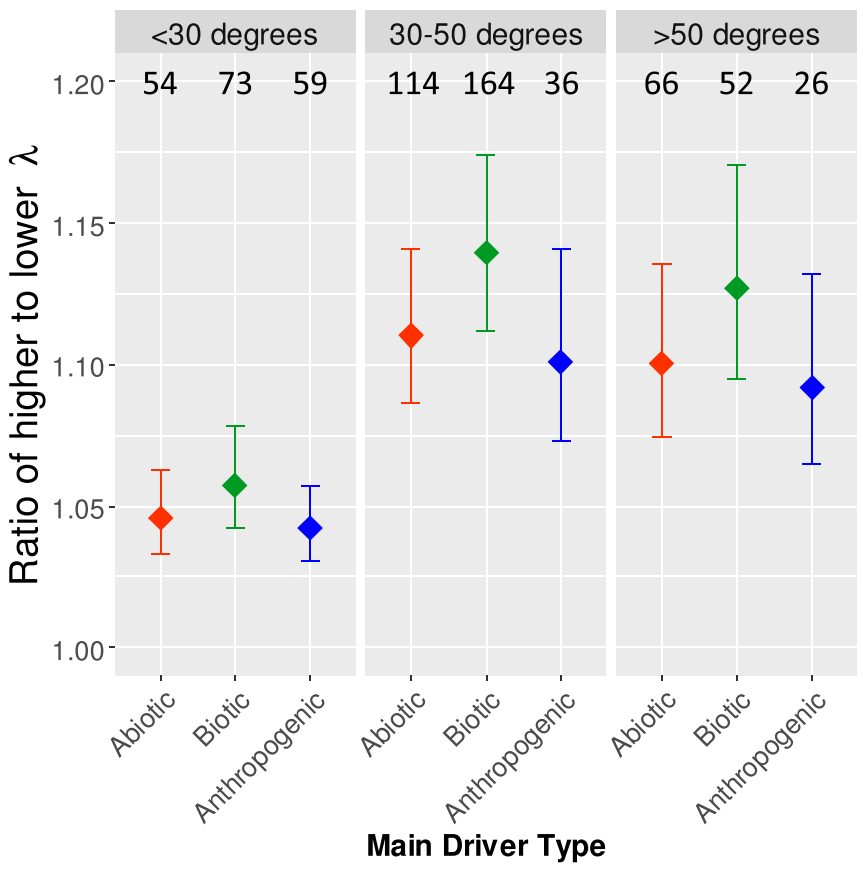

Fig. 4. Effects of main driver types on $\lambda$ in 3 latitudinal zones (distance in degrees $\mathrm{N}$ or $\mathrm{S}$ of the equator); points, error bars, and numerals at Top as in Fig. 1.

interactions may influence distributions, the lion's share of work to predict how climate change will shift distributions has used species distribution models (SDMs), which typically use only climate variables (less often nonclimate abiotic variables, and only very rarely biotic variables) as predictors of species' occurrences (refs. 12 and 13, but see refs. 14 and 15). However, if the influence of biotic interactions is substantial, even if lower on average than the influence of climate and other abiotic drivers, then changes in the distributions and abundances of interacting species, which may reflect indirect effects of climate change, species introductions, or other environmental changes, may be just as important to weigh as the direct effects of climate change per se. While this warning has often been made in the ecological literature, our finding that the average effect magnitude of biotic drivers is substantial relative to abiotic drivers makes this warning more poignant, and suggests that changes in biotic drivers cannot be safely ignored, unless we know that biotic and abiotic drivers are tightly correlated and will continue to be so as the environment changes.

While the major driver types exerted similar effects on average, we did see significant differences among driver subtypes (Fig. $1 A$ ). Interestingly, within the class of abiotic drivers, climate was less influential than was disturbance (which in our database was mainly fire). Indirect effects of climate change on disturbance frequency (e.g., a reduction in precipitation leading to an increase in fire frequency) or alteration of disturbance by humans may therefore be more important to consider than direct effects of climate change per se (and few SDMs consider disturbance). We also note that climate and natural enemies (primarily herbivores) received the most attention in the studies in our database among the abiotic and biotic drivers, respectively (see numbers at Top of Fig. 1A), but neither were the most influential drivers within its class, suggesting a potential value in altering future research priorities to improve projections of environmental change responses.

A second important implication of our results is that the effect magnitudes of abiotic, biotic, and anthropogenic drivers defy simple hypotheses about when and where one should be stronger than the others. Some evolutionary biologists have argued that biotic forces should impose stronger selection than abiotic forces over the long run because interacting species are also undergoing evolutionary change (16-18), but others have argued that abiotic factors have been more influential as past agents of selection (19), or that humans impose the strongest current impact (ref. 20, but see ref. 21). Darwin (10) argued that biotic forces (in particular, competition and natural enemies) should increase in importance relative to abiotic forces as the low latitude or abiotically more benign range limit of a species is approached, but decrease in importance toward the high latitude or more abiotically harsh range limit. Our results indicate that both abiotic and biotic forces are influential, both in abiotically benign (e.g., at low latitudes and in high NPP biomes) and in seemingly more challenging environments. It may be that latitude and biome are not good proxies for harshness of the abiotic environment, and that a more nuanced treatment of harshness is necessary (as in ref. 11). However, assuming our results hold, perhaps the necessary first step of evolving the ability to tolerate harsh abiotic conditions, both in plants and in interacting species, then sets the stage for biotic forces to become as influential on fitness as are abiotic forces. Similarly, abiotic stress in harsh environments may have preadapted plants to anthropogenic stress, leading to similar effects of all 3 main driver types in such environments. However, interestingly, we did find that plants at middle and high latitudes (Fig. 4) and in less productive biomes (Fig. 5) were more sensitive to drivers of all 3 main types, suggesting that more marginal habitats may increase the influence of all drivers. In contrast, a woody growth form may make plants more resilient on average to changes in all types of drivers (Fig. 3).

A third important implication of our findings is that, because the strength of the impacts of anthropogenic drivers rivals those of abiotic and biotic drivers, we must always consider the possibility that changes in the ways that humans use or manage landscapes will modify or even overwhelm the effects of changes in climate. We did find that anthropogenic drivers had a weaker effect on woody plants than on herbaceous plants (Fig. 2). However, this result is likely due to the fact that harvesting studies of

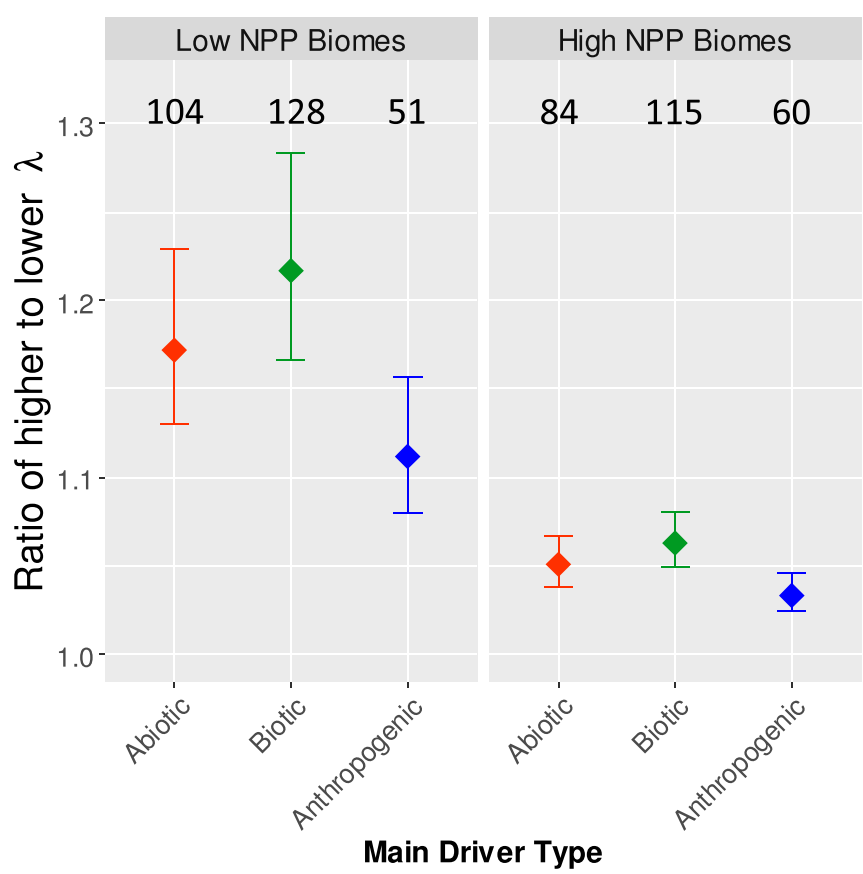

Fig. 5. Effects of main driver types on $\lambda$ in low NPP vs. high NPP biomes (a measure of the overall harshness of the abiotic environment). Studies performed north of $55^{\circ} \mathrm{N}$ were excluded, as all came from low NPP biomes (see text); points, error bars, and numerals at Top as in Fig. 1. 
herbaceous plants frequently included lethal harvest of entire plants, but few studies of woody plants did (see SI Appendix). In addition, our database does not include complete destruction of entire populations by humans, as occurs, for example, in conversion of forest to agriculture. Widespread anthropogenic habitat destruction means that effects of humans on biodiversity are certainly even greater than what we show here.

Fourth, that no one driver type (abiotic, biotic, or anthropogenic) is overwhelmingly more influential has 3 important evolutionary implications. First, as all of our estimates of driver effects are based on total fitness (i.e., $\lambda$ ), they may more accurately indicate the importance of potential selective agents than would certain fitness components that might weakly translate into total fitness (e.g., fecundity in long-lived plants). The great majority of the selection estimates in recent metaanalyses (e.g., $>98 \%$ in refs. 22 and 23) were based upon fitness components rather than total fitness. Second, selection on traits that modulate the fitness effects of the driver should be substantial on average for all of the main driver types. For example, traits that reduce negative effects of climate, traits that reduce herbivory or its impacts, and traits that compensate for harvesting effects should on average experience selection strengths that do not differ greatly. Third, because $\lambda$ measures mean absolute (not relative) fitness, effects of a driver on $\lambda$ can alter selection strength even when the driver has equivalent effects on all genotypes, because it affects the variance in relative fitness among genotypes (the so-called "opportunity for selection") (21, 23-26). Because the magnitudes of the effects of abiotic, biotic, and anthropogenic drivers on mean absolute fitness (and thus on the opportunity for selection) are similar, their indirect effects on the strength of selection on traits that do not directly modulate the impacts of those drivers are also likely to be of a similar order of magnitude (SI Appendix). Caruso et al. (23) found that experimental manipulation of biotic and abiotic factors had equivalent effects on the strength of trait selection, and they showed that selection was stronger when mean absolute fitness was lower, implicating a change in the opportunity for selection. Similarly, Fugère and Hendry (21) argued that anthropogenic disturbance indirectly weakened selection on traits by increasing mean absolute fitness (presumably because the benefits of disturbance outweighed its costs).

Future comparative studies of the effects of different types of drivers on plant population growth and fitness would benefit if demographers measured driver levels along with vital rates (3). For most $(57.8 \%)$ of the $|L|$ values we used to measure the magnitudes of driver effects, the source papers did not specify an actual level of the driver associated with the $2 \lambda$ values, and a majority of the others did not measure the driver mean and SD, thus making it impossible to assess how the difference in driver levels compares to the average and range of variation the plant population might normally experience. This lack of information also limits our ability to extrapolate $\lambda$ to future driver levels. In addition, most of the original studies did not account for effects of intraspecific density on $\lambda$ values; relaxation of negative density dependence when driver levels were unfavorable may have reduced driver impacts on $\lambda$ (27). Therefore, to improve upon the analyses we have presented here, the next generation of demographic studies, both observational and experimental, should invest more effort to actually measure levels of putative drivers and to account for the joint effects of drivers and intraspecific density (for a good example of both, see ref. 28).

In conclusion, our intention in using the best available information to evaluate effects of drivers on plant population growth and fitness was to look both backward and forward in time. Looking backward, we find no evidence to conclude that abiotic forces have been dramatically more or less influential than biotic forces in shaping the plant traits, abundances, and geographical distributions that we see today. Looking forward, our findings make it clear that we must simultaneously account for abiotic, biotic, and (increasingly, given growth in human population size and per-capita resource consumption) anthropogenic forces if we are to accurately forecast the ecological and evolutionary futures of terrestrial plants (and thus ecosystem services such as carbon sequestration in which they play a disproportionate global role).

\section{Methods}

We began with the list of papers reviewed by Ehrlén et al. (29), supplemented by a Web of Science search to identify papers published before November 30,2017 , that examined how $\lambda$ for plant populations responded to drivers (see search terms in SI Appendix), and supplemented the search results with other papers of which we knew (see list of papers and Dataset S1). To be included in our analysis, papers had to 1) use a structured population model (projection matrix model, integral projection model, or individual-based model) to compute a deterministic or stochastic $\lambda$ (typically asymptotic, but sometimes transient), and 2) present estimates of $\lambda$ for different levels of an identified driver (or present information such as projection matrices that allowed us to compute the $\lambda$ values ourselves). We excluded studies that did not attempt to identify the specific driver that might have been responsible for any differences in $\lambda$ (e.g., those that compared different habitats); were performed in row-crop agriculture (e.g., studies of weed demography); or used hypothetical perturbations of the model to examine potential driver impacts, rather than quantifying actual driver impacts on vital rates (most of these were studies of logging effects on tree populations). We included both experimental studies, in which the investigators manipulated levels of the driver, and observational studies, in which the investigators quantified $\lambda$ at different driver levels experienced by the field populations.

We extracted (by digitizing graphs if necessary) 2 estimates of $\lambda$, one at the highest and one at the lowest level of the driver examined, for each driver and population in each paper. For exclusion experiments, such as neighbor removal or herbivore caging experiments, these represented $\lambda$ in the presence and absence of the driver, and many observational studies presented $\lambda$ values at only 2 driver levels. Other studies performed a linear regression of $\lambda$ against measured levels of the driver, combining $\lambda$ and driver levels across populations or subpopulations, or across years within populations. For those studies, we extracted the predicted $\lambda$ values from the regression line at the highest and lowest levels of the driver observed in the study. Some studies (particular those in which the driver was disturbance return interval) documented a nonlinear relationship between $\lambda$ and the driver; for these studies, we took the highest and lowest level of $\lambda$ from the fitted curve across all levels of the driver (even if these occurred at an intermediate driver level). Because linear and nonlinear regressions include more information about the $\lambda$-driver relationship than do simple pairs of $\lambda$ values from 2 levels of the driver (whether experimentally manipulated or unmanipulated), we assigned more weight in our analyses to the regression-based estimates (specifically, we used as weights the regression sample size for the regression estimates and a value of 2 for $\lambda$ pairs).

We used the pair of $\lambda$ values to compute the log response ratio:

$$
L=\log \left(\lambda_{\text {low }} / \lambda_{\text {high }}\right),
$$

where $\lambda_{\text {high }}$ and $\lambda_{\text {low }}$ are the population growth rates at high and low levels of the driver, respectively. Use of the ratio assumes drivers affect $\lambda$ multiplicatively, which is reasonable given that $\lambda$ must be nonnegative. For drivers that reduce plant population growth rate (e.g., competitors, herbivores), $\lambda_{\text {high }}<\lambda_{\text {low }}$ and $L<0$, whereas for beneficial drivers, $L>0$. However, for our purpose here of comparing the relative impacts of different types of drivers, we are not interested in the sign of $L$ but only its magnitude; hence we analyzed the absolute value of $L,|L|$ (Eq. 1). In addition, because the use of the absolute value causes right skew in our estimates of effect sizes, we logtransformed the $|L|$ values prior to statistical analysis to improve normality. If a study reported multiple yearly values of $|L|$ for the same driver and population based on single-year $\lambda$ values (i.e., the dominant eigenvalues of 1-y projection matrices), we included only the among-year average of those $|L|$ values. For factorial studies that crossed levels of 2 or more drivers, we included an $|L|$ value for a given driver at each level of the other driver(s). All response and predictor variables used in our analysis are given in Dataset S2 (9).

Fewer than half of the papers we reviewed presented SEs of the $\lambda$ values they reported, and the diverse approaches we used to obtain $\lambda_{\text {high }}$ and $\lambda_{\text {low }}$ values further precluded a formal metaanalysis (30). In addition to the effect of sampling variation on the precision of the $\lambda$ estimates, measures of the magnitude of an effect, such as $|L|$, will be upwardly biased by sampling variation (30). However, we see no reason to expect that sampling variation, and thus bias, should be greater for one type of driver than another. Given 
the lack of SEs, we performed an informal metaanalysis $(21,30)$. Specifically, we tested for differences in the effects of different types of drivers, and their interactions with other factors such as biome, latitude, and plant growth form, using linear mixed models [Imer package in R (31)], including the publication as a random factor to account for any possible methodological nonindependence between different response values from the same study.

We assessed whether relative driver impacts differed between plants of different growth form (woody vs. herbaceous) via the statistical significance of the growth form $\times$ driver type interaction. We assessed Darwin's hypothesis by assigning each $|L|$ to 1 of 3 zones, based on degrees of latitude of the study site away from the equator: $<30,30$ to 50 , and $>50^{\circ}$ (all of the latter were in the northern hemisphere), and testing for a driver type $x$ latitudinal zone interaction. These zones were chosen to retain a reasonable sample size of $|L|$ values in each zone while still separating tropical/subtropical and boreal/arctic study locations from temperate ones. To evaluate whether relative driver type impacts differed between biomes with more vs. less harsh abiotic conditions, we divided studies into 2 groups, depending on whether the biome in which the study was performed has an average annual NPP $\leq$ or $>1 \mathrm{~kg} \mathrm{C} / \mathrm{m}^{2} / \mathrm{y}$, according to ref. 32. Because NPP will be low when either growing season temperature or annual precipitation is low, it integrates 2 components of abiotic harshness. All data points from $>55^{\circ} \mathrm{N}$ came from low NPP biomes (boreal forest, northern grassland, and arctic/ alpine tundra); thus to reduce the potential to confound our analyses of latitudinal patterns and of abiotic harshness more broadly, we excluded those high-latitude points from the biome comparison. The resulting low NPP biomes included alpine tundra, deserts, grasslands, and shrublands, while high NPP biomes included temperate and tropical forests and savannahs. The

1. M. C. Urban et al., Improving the forecast for biodiversity under climate change. Science 353, aad8466 (2016).

2. B. J. Cardinale et al., Biodiversity loss and its impact on humanity. Nature 486, 59-67 (2012).

3. H. R. Pulliam, On the relationship between niche and distribution. Ecol. Lett. 3, 349361 (2000).

4. R. D. Holt, Bringing the Hutchinsonian niche into the 21st century: Ecological and evolutionary perspectives. Proc. Natl. Acad. Sci. U.S.A. 106 (suppl. 2), 19659-19665 (2009).

5. J. Ehrlén, W. F. Morris, Predicting changes in the distribution and abundance of species under environmental change. Ecol. Lett. 18, 303-314 (2015).

6. B. Charlesworth, Evolution in Age-Structured Populations (Cambridge University Press, New York, ed. 2, 1994)

7. R. Lande, A quantitative genetic theory of life-history evolution. Ecology 63, 607-615 (1982).

8. H. Caswell, Matrix Population Models: Construction, Analysis, and Interpretation (Sinauer Associates, Sunderland, MA, ed. 2, 2001).

9. W. Morris, Biotic and anthropogenic forces rival climatic/abiotic factors in determining global plant population growth and fitness. Dryad. https://doi.org/10.5061/dryad.95x69p8fm. Deposited 13 December 2019.

10. C. Darwin, The Origin of Species (John Murray, London, UK, 1859)

11. A. M. Louthan et al., Aridity weakens population-level effects of multiple species interactions on Hibiscus meyeri. Proc. Natl. Acad. Sci. U.S.A. 115, 543-548 (2018)

12. J. Franklin, Mapping Species Distributions: Spatial Inference and Predictions (Cambridge University Press, Cambridge, UK, 2009).

13. A. T. Peterson et al., Ecological Niches and Geographical Distributions (Princeton University Press, Princeton, NJ, 2011).

14. M. S. Wisz et al., The role of biotic interactions in shaping distributions and realised assemblages of species: Implications for species distribution modelling. Biol. Rev. Camb. Philos. Soc. 88, 15-30 (2013).

15. W. D. Kissling et al., Towards novel approaches to modelling biotic interactions in multispecies assemblages at large spatial extents. J. Biogeogr. 39, 2163-2178 (2012).

16. R. A. Fisher, The Genetical Theory of Natural Selection (Oxford University Press, Oxford, UK, 1930). database contained too few estimates of $|L|$ from extreme biomes (deserts and alpine tundra) to reliably compare only those biomes to less extreme ones (Dataset S1).

When the original studies presented the actual levels of the driver $d_{\text {high }}$ and $d_{\text {low }}$ that produced $\lambda_{\text {high }}$ and $\lambda_{\text {low }}$, respectively, as well as the mean $\bar{d}$ and SD $S D_{d}$ of the driver across space and/or time (based on at least 3 data points), we assessed whether max $\left(\left|d_{\text {high }}-\bar{d}\right| / S D_{d}, d_{\text {low }}-\bar{d} / S D_{d}\right)$ differed between abiotic and biotic drivers using a linear mixed model with publication as a random factor and weighting each value of the dependent variable by the number of driver values used to estimate $\bar{d}$ and $S D_{d}$. We used a similar weighted linear mixed model to test for differences in scaled sensitivity $|S|$ (Eq. 2) between abiotic and biotic drivers. Both the maximum driver deviation and the scaled sensitivities were log-transformed to improve normality.

Data Availability. All of the data extracted from the published studies that were used to perform the analyses in this paper are available in Datasets S1 and S2, and are archived on Dryad (DOI: 10.5061/dryad.95x69p8fm).

ACKNOWLEDGMENTS. We thank the many plant demographers whose published studies made the analysis we present here possible. Research was supported by NSF Grants DEB 1242558 and 1753980; Strategic Environmental Research and Development Program Contract W912HQ18C0101; and a grant from the Swedish Science Council (to W.F.M.). J.E. and J.P.D. acknowledge funding from the Swedish Research Council for Environment, Agricultural Sciences, and Spatial Planning (FORMAS), and J.P.D. acknowledges funding from the Independent Research Fund Denmark.

17. L. Van Valen, A new evolutionary law. Evol. Theory 1, 1-30 (1973)

18. K. L. Voje, O. H. Nolen, L. H. Liow, N. C. Stenseth, The role of biotic forces in driving macroevolution: Beyond the Red Queen. Proc. R. Soc. B Biol. Sci. 282, 20150186 (2015).

19. A. D. Barnosky, Distinguishing the effects of the Red Queen and court jester on meiocene mammal evolution in the northern rocky mountains. J. Vertebr. Paleontol. 21, 172-185 (2001)

20. S. R. Palumbi, Humans as the world's greatest evolutionary force. Science $293,1786-$ 1790 (2001).

21. V. Fugère, A. P. Hendry, Human influences on the strength of phenotypic selection Proc. Natl. Acad. Sci. U.S.A. 115, 10070-10075 (2018)

22. A. M. Siepielski et al., Precipitation drives global variation in natural selection. Science 355, 959-962 (2017)

23. C. M. Caruso et al., What are the environmental determinants of phenotypic selection? A meta-analysis of experimental studies. Am. Nat. 190, 363-376 (2017).

24. J. F. Crow, Some possibilities for measuring selection intensities in man. Hum. Biol. 30, 1-13 (1958).

25. H. D. Rundle, S. M. Vamosi, Selection may be strongest when resources are scarce: A comment on Wilson. Evol. Ecol. 10, 559-563 (1996)

26. D. C. Hunter, J. M. Pemberton, J. G. Pilkington, M. B. Morrissey, Quantification and decomposition of environment-selection relationships. Evolution 72, 851-866 (2018).

27. J. P. Dahlgren, H. Ostergård, J. Ehrlén, Local environment and density-dependent feedbacks determine population growth in a forest herb. Oecologia 176, 10231032 (2014).

28. J. M. Diez, I. Giladi, R. Warren, H. R. Pulliam, Probabilistic and spatially variable niches inferred from demography. J. Ecol. 102, 544-554 (2014).

29. J. Ehrlén, W. F. Morris, T. von Euler, J. P. Dahlgren, Advancing environmentally explicit structured population models of plants. J. Ecol. 104, 292-305 (2016)

30. M. B. Morrissey, Meta-analysis of magnitudes, differences and variation in evolutionary parameters. J. Evol. Biol. 29, 1882-1904 (2016)

31. R Computing Team, $R$ : A Language and Environment for Statistical Computing ( $R$ Foundation for Statistical Computing, Vienna, Austria, 2017).

32. B. Saugier, J. Roy, H. A. Mooney, "Estimations of global terrestrial productivity: Converging toward a single number?" in Terrestrial Global Productivity, J. Roy, B. Saugier, H. A. Mooney, Eds. (Academic Press, San Diego, CA, 2001), pp. 543-557. 